\title{
Dopamine D2-Like Receptors Modulate Intrinsic Properties and Synaptic Transmission of Parvalbumin Interneurons in the Mouse Primary Motor Cortex
}

\author{
Jérémy Cousineau, ${ }^{1,2^{\star}}$ Léa Lescouzères, ${ }^{1,2,3^{*}}$ Anne Taupignon, ${ }^{1,2}$ Lorena Delgado-Zabalza, ${ }^{1,2,4}$ \\ Emmanuel Valjent, ${ }^{5}$ Jérôme Baufreton, ${ }^{1,2}$ and Morgane Le Bon-Jégo ${ }^{1,2}$
}

https://doi.org/10.1523/ENEURO.0081-20.2020

${ }^{1}$ Université de Bordeaux, Institut des Maladies Neurodégénératives, Unité Mixte de Recherche 5293, Bordeaux F33000 , France, ${ }^{2}$ Centre National de la Recherche Scientifique, Institut des Maladies Neurodégénératives, Unité Mixte de Recherche 5293, Bordeaux F-33000, France, ${ }^{3}$ Institute for Neuroscience of Montpellier, Institut National de la Santé et de la Recherche Médicale, University of Montpellier, Montpellier 34091, France, ${ }^{4}$ University of the Basque Country (Universidad del País Vasco/Euskal Herriko Unibertsitatea), Leioa 48940, Spain, and ${ }^{5}$ Institut de Génomique Fonctionnelle, Centre National de la Recherche Scientifique, Institut National de la Santé et de la Recherche Médicale, University of Montpellier, Montpellier 34094, France

\begin{abstract}
Dopamine (DA) plays a crucial role in the control of motor and higher cognitive functions such as learning, working memory, and decision making. The primary motor cortex (M1), which is essential for motor control and the acquisition of motor skills, receives dopaminergic inputs in its superficial and deep layers from the midbrain. However, the precise action of DA and DA receptor subtypes on the cortical microcircuits of M1 remains poorly understood. The aim of this work was to investigate in mice how DA, through the activation of D2-like receptors (D2Rs), modulates the cellular and synaptic activity of M1 parvalbumin-expressing interneurons (PVINs) which are crucial to regulate the spike output of pyramidal neurons (PNs). By combining immunofluorescence, ex vivo electrophysiology, pharmacology and optogenetics approaches, we show that D2R activation increases neuronal excitability of PVINs and GABAergic synaptic transmission between PVINs and PNs in Layer V of M1. Our data reveal how cortical DA modulates M1 microcircuitry, which could be important in the acquisition of motor skills.
\end{abstract}

Key words: D2 receptors; electrophysiology; neuromodulation; parvalbumin interneuron; primary motor cortex

\section{Significance Statement}

Primary motor cortex (M1), which is a region essential for motor control and the acquisition of motor skills, receives dopaminergic inputs from the midbrain. However, precise action of dopamine (DA) and its receptor subtypes on specific cell types in M1 remained poorly understood. Here, we demonstrate in M1 that DA D2like receptors (D2Rs) are present in parvalbumin interneurons (PVINs) and their activation increases the excitability of the PVINs, which are crucial to regulate the spike output of pyramidal neurons (PNs). Moreover, the activation of the D2R facilitates the GABAergic synaptic transmission of those PVINs on Layer V PNs. These results highlight how cortical DA modulates the functioning of M1 microcircuit which activity is disturbed in hypodopaminergic and hyperdopaminergic states.

Received March 4, 2020; accepted March 10, 2020; First published April 13, 2020.

The authors declare no competing financial interests.
Author contributions: M.L.B.-J. designed research; J.C., L.L., A.T., L.D.-Z., E.V., J.B., and M.L.B.-J. performed research; J.C., L.L., A.I.T., E.V., J.B., and M.L.B.-J. analyzed data; A.I.T., E.V., J.B., and M.L.B.-J. wrote the paper. 


\section{Introduction}

The neuromodulator dopamine (DA) plays a key role in the ability of neural circuits to adaptively control behavior (Schultz, 2007; Vitrac and Benoit-Marand, 2017; Berke, 2018). Indeed, the DA system plays a major role in motor and cognitive functions through its interactions with several brain regions, and its dysregulation leads to cognitive dysfunction (Duvarci et al., 2018) and pathologies like Parkinson's disease and schizophrenia (Nieoullon, 2002). Recently, it has been suggested that the primary motor cortex (M1) may also be influenced by DA (Hosp and Luft, 2013; Guo et al., 2015). The architecture of the dopaminergic inputs to $\mathrm{M} 1$ has been well characterized anatomically mainly in rodent and primate. Coming mainly from the ventral tegmental area (VTA) but also from the substantia nigra pars compacta (SNc), they richly innervate the superficial and deep layers of M1 (Descarries et al., 1987; Lewis et al., 1987; Vitrac et al., 2014; Hosp et al., 2015). However, their functional significance is poorly understood and reports of their effects remain conflicting, presumably because of the in vivo exploration and wide neuronal diversity in M1 (Hosp and Luft, 2013; Vitrac et al., 2014; Vitrac and Benoit-Marand, 2017).

DA acts via two main classes of receptors, the D1-like (D1R) and the D2-like (D2R) family, which differentially modulate adenylyl cyclase (Beaulieu and Gainetdinov, 2011). In M1, both families of DA receptors are present in the deep layers (Dawson et al., 1986; Lidow et al., 1989; Weiner et al., 1991; Gaspar et al., 1995). Based on in situ hybridization, it appears that Layer $\mathrm{V}$ of the cortex, the layer where pyramidal neurons (PNs) integrate inputs from many sources and distribute information to cortical and subcortical structures, mainly contains D2R mRNA (Gaspar et al., 1995).

Previous work in rats has described the effect of DA on neuronal activity $\mathrm{M} 1$ neurons in vivo, but most of these studies focused on PNs and draw different conclusions regarding an inhibitory or excitatory effect of DA on neuronal activity in M1 (Awenowicz and Porter, 2002; Vitrac et al., 2014). However, there is a large body of evidence supporting that inhibition is important in controlling the excitatory circuits. Among the various interneurons (INs; Ascoli et al., 2008; DeFelipe et al., 2013; Lodato et al., 2015; Markram et al., 2015), parvalbumin-expressing INs (PVINs) represent a minority cell type. However, they are

This work was supported by Centre National de la Recherche Scientifique, University of Bordeaux, Institut National de la Santé et de la Recherche Médicale, Fondation pour la Recherche Médicale (FRM), and the French National Research Agency Grant ANR-DOPAFEAR (to E.V.). J.C. received a Ph.D. fellowship from the FRM (ECO201806006853, Fondation Yolande Calvet).

This article was first published as a preprint and is available at: https://www. biorxiv.org/content/10.1101/802140v1.

*J.C. and L.L. contributed equally to this work.

Correspondence should be addressed to Morgane Le Bon-Jégo at morgane .jego@u-bordeaux.fr.

https://doi.org/10.1523/ENEURO.0081-20.2020

Copyright (C) 2020 Cousineau et al.

This is an open-access article distributed under the terms of the Creative Commons Attribution 4.0 International license, which permits unrestricted use, distribution and reproduction in any medium provided that the original work is properly attributed. crucial for normal brain function (Donato et al., 2013; Courtin et al., 2014): they powerfully regulate the spike output of PNs, mainly by targeting their somatic and perisomatic regions (Hu et al., 2014). In addition, they are also recruited for motor execution (Estebanez et al., 2017).

To better understand the cellular and network basis of DA action in $M 1$, it is necessary to determine the cellular targets of DA innervation. We hypothesized that DA in M1 contributes to normal microcircuit processing by modulating the activity of PVINs in Layer V through D2R. To test this hypothesis, we performed qualitative mapping of the M1 neuronal population expressing D2R and electrophysiologically characterized these D2R-positive neurons. Then, we investigated the impact of D2R activation on the excitability of PVINs using patch-clamp electrophysiology and on GABAergic synaptic transmission between PVINs and PNs using optogenetics.

We found that D2Rs are broadly expressed in $M 1$, in both superficial and deep layers. In Layer V, the majority of neurons expressing D2R are PVINs. Moreover, D2R agonists increase the excitability of PVINs and also enhance GABAergic synaptic transmission between PVINs and PNs. Our results clarify and highlight the role of DA in modulating the activity of cortical microcircuits in M1.

\section{Animals}

All experiments were performed in accordance with the guidelines of the French Agriculture and Forestry Ministry for handling animals (authorization number/license D34-172-13 and APAFIS \#14 255). C57BL6J and three transgenic mouse lines were used for this study. Drd2-Cre:Ribotag mice were used for the morphologic study (Puighermanal et al., 2015). PV-Cre:Ai9T mice were generated by crossing PV-Cre mice (B6;129P2$P_{\text {Valb }}^{\text {tm1(cre)Arbr } / J ; ~ J A X ~ s t o c k ~ \# 008069 ; ~ K a i s e r ~ e t ~ a l ., ~ 2016) ~}$

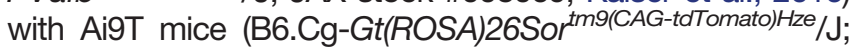
stock \#007909) and the Drd2-Cre:Ai9T line was generated by crossing Drd2-Cre mice (Tg(Drd2-Cre)ER44Gsat; Gensat Project at Rockefeller University) with Ai9T mice (B6.Cg-Gt

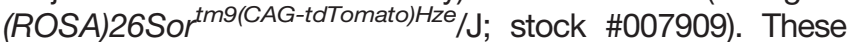
two lines express the red fluorescent protein double-tomato (tdTom) under endogenous regulatory elements of the parvalbumin gene locus and those of D2R, respectively. Males and females, 8-12 weeks old, were used for ex vivo experiments. All animals were maintained in a 12/12 h light/dark cycle, in stable conditions of temperature and humidity, with access to food and water ad libitum.

\section{Tissue preparation and immunofluorescence Mapping of the distribution of $D 2 R$}

Male Drd2-Cre:Ribotag mice, 8-10 weeks old $(n=6)$, were used for the morphologic study. Mice were rapidly anesthetized with Euthasol (360 mg/kg, i.p.; Laboratoire TVM) and transcardially perfused with $4 \%$ (w/v) paraformaldehyde in $0.1 \mathrm{M}$ sodium phosphate buffer ( $\mathrm{pH} 7.5)$. Brains were postfixed overnight in the same solution and stored at $4^{\circ} \mathrm{C}$. Sections of $30 \mu \mathrm{m}$ were cut with a vibratome (Leica) and stored at $-20^{\circ} \mathrm{C}$ in a solution containing $30 \%(\mathrm{v} / \mathrm{v})$ ethylene glycol, 30\% (v/v) glycerol, and $0.1 \mathrm{M}$ 
Table 1: List of primary antibodies for the mapping of the distribution of D2R

\begin{tabular}{|c|c|c|c|c|}
\hline Antigen & Host & Dilution & Supplier & Catalog no. \\
\hline $\mathrm{HA}$ & Mouse & $1: 1000$ & Covance & MMS101R \\
\hline CR & Rabbit & $1: 1000$ & Swant & CR7699/3H \\
\hline Parvalbumin & Rabbit & $1: 1000$ & Swant & PV25 \\
\hline NPY & Rabbit & $1: 500$ & Abcam & \#ab10980 \\
\hline nNOS & Sheep & $1: 3000$ & Gift from Dr. V. Prevot (Herbison et al., 1996) & \\
\hline
\end{tabular}

sodium phosphate buffer until they were processed for immunofluorescence. M1 sections were identified using a mouse brain atlas; sections located between +1.60 and $+0.98 \mathrm{~mm}$ from bregma were included in the analysis (Franklin and Paxinos, 2007). Sections were processed as follows: free-floating sections were rinsed $3 \times 10 \mathrm{~min}$ in Tris-buffered saline (TBS; $50 \mathrm{~mm}$ Tris- $\mathrm{HCl}$ and $150 \mathrm{~mm}$ $\mathrm{NaCl}, \mathrm{pH} 7.5)$. After 15-min incubation in 0.2\% (v/v) Triton $X-100$ in TBS, sections were rinsed again in TBS and blocked for $1 \mathrm{~h}$ in a solution of $3 \%$ BSA in TBS. Finally, they were incubated $72 \mathrm{~h}$ at $4^{\circ} \mathrm{C}$ in $1 \% \mathrm{BSA}, 0.15 \%$ Triton $\mathrm{X}-100$ with the primary antibodies (Table 1). Sections were rinsed $3 \times 10 \mathrm{~min}$ in TBS and incubated for $45 \mathrm{~min}$ with goat Cy2- and Cy3-coupled (1:500, Jackson ImmunoResearch) and/or goat Alexa Fluor 488 (1:500, Life Technologies). Sections were rinsed $2 \times 10 \mathrm{~min}$ in TBS and twice in Tris-buffer (1 $\mathrm{M}, \mathrm{pH}$ 7.5) before mounting in DPX (Sigma-Aldrich). Confocal microscopy and image analysis were conducted at the Montpellier RIO Imaging Facility. All images covering the $\mathrm{M} 1$ region were single confocal sections acquired using sequential laser scanning confocal microscopy (Leica SP8) and stitched together as a single image. Double-labeled images from each region of interest were also single confocal sections obtained using sequential laser scanning confocal microscopy (Leica SP8). Hemagglutinin (HA)-immunopositive cells were pseudo-colored cyan and other immunoreactive markers were pseudo-colored orange. Images used for quantification were all single confocal sections. HA-positive cells were manually counted using the cell counter plugin of the ImageJ software in M1, taking into account the cortical layers (Layer I, Layers II-III, and Layers $\mathrm{V}-\mathrm{VI}$ ). Adjacent serial sections were never counted for the same marker to avoid any double counting of hemisected neurons. Values in the histograms in Figure $1 B$ represent the percentage of $\mathrm{HA}$-expressing neurons in Layer I, Layers II-III, and Layers V-VI ( $n=5-6$ mice). Total numbers of HA- and marker-positive cells counted are indicated between parentheses.

\section{Slice preparation}

Coronal sections containing M1 were prepared from 8to 12 -week-old mice. Mice were first sedated by inhaling isoflurane (4\%) for $\sim 30 \mathrm{~s}$ and then deeply anesthetized with a mixture of ketamine and xylazine (100 and $20 \mathrm{mg} /$ $\mathrm{kg}$, i.p., respectively). After the disappearance of the reflexes, a thoracotomy was performed to allow transcardial perfusion of a saturated $\left(95 \% \mathrm{O}_{2} / 5 \% \mathrm{CO}_{2}\right)$ ice-cold solution containing $250 \mathrm{~mm}$ sucrose, $10 \mathrm{~mm} \mathrm{MgSO}_{4} \cdot 7 \mathrm{H}_{2} \mathrm{O}, 2.5$ $\mathrm{mm} \mathrm{KCl}, 1.25 \mathrm{~mm} \mathrm{NaH} \mathrm{PO}_{4} \cdot \mathrm{H}_{2} \mathrm{O}, 0.5 \mathrm{~mm} \mathrm{CaCl}{ }_{2} \cdot \mathrm{H}_{2} \mathrm{O}, 1.3$ $\mathrm{mm} \mathrm{MgCl}_{2}, 26 \mathrm{~mm} \mathrm{NaHCO}_{3}$, and $10 \mathrm{~mm}$ D-glucose. After decapitation, each brain was quickly removed and cut into coronal slices $(300-350 \mu \mathrm{m})$ using a vibratome (VT-

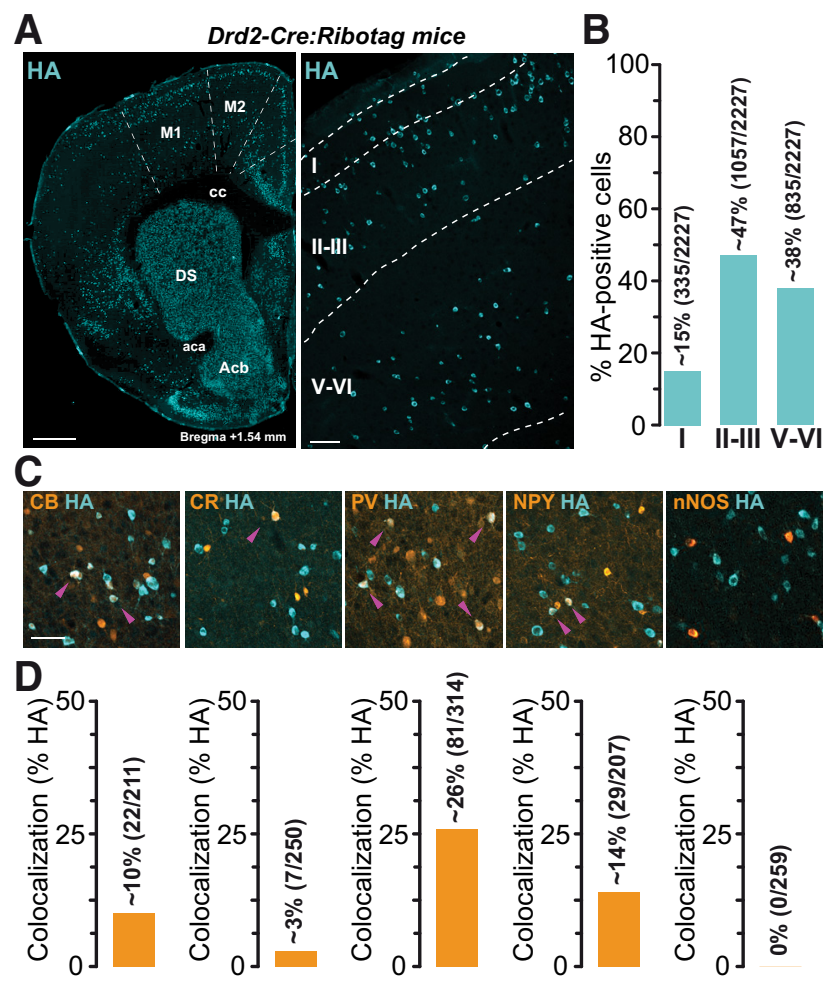

Figure 1. Distribution of D2R-expressing neurons in $M 1$ in Drd2-Cre:Ribotag mice. A, Coronal section from Drd2-Cre: Ribotag mice stained with hemagglutinin $(\mathrm{HA})$ showing the distribution of D2R-expressing neurons in the different layers of M1. Scale bars: $500 \mu \mathrm{m}$ (left) and $50 \mu \mathrm{m}$ (right). B, Histogram showing the distribution of HA-labeled neurons in Layer I, Layers II-III, and Layers V-VI of the M1 (17 hemispheres analyzed, 5 mice). The distribution is expressed as a percentage of HA-positive neurons in all layers. The number of HA-positive cells counted is indicated between parentheses. $\boldsymbol{C}, \mathrm{HA}$ (cyan) and calbindin-D28k (CB), Calretinin (CR), parvalbumin (PV), neuropeptide $Y$ (NPY), and nNOS (orange) immunofluorescence in M1 Layers V-VI of Drd2-Cre:Ribotag mice. Magenta arrowheads indicate HA/markers-positive neurons. Scale bars: $40 \mu \mathrm{m}$. $\boldsymbol{D}$, Histograms showing the co-expression as a percentage of HA-positive cells in M1 Layers V-VI of Drd2-Cre:Ribotag mice (blue, left). The total numbers of HA- and marker-positive cells counted are indicated between parentheses. DS: dorsal striatum; cc: corpus callosum; Acb: nucleus accumbens; aca: anterior commissure. 
1200S; Leica Microsystems). The slices were then incubated at $34^{\circ} \mathrm{C}$ for $1 \mathrm{~h}$ in a standard artificial CSF (ACSF) saturated by bubbling $95 \% \mathrm{O}_{2} / 5 \% \mathrm{CO}_{2}$ and containing $126 \mathrm{~mm} \mathrm{NaCl}, 2.5 \mathrm{~mm} \mathrm{KCl}, 1.25 \mathrm{~mm} \mathrm{NaH}_{2} \mathrm{PO}_{4} \cdot \mathrm{H}_{2} \mathrm{O}, 2 \mathrm{~mm}$ $\mathrm{CaCl}_{2} \cdot \mathrm{H}_{2} \mathrm{O}, 2 \mathrm{~mm} \mathrm{MgSO} \cdot 7 \mathrm{H}_{2} \mathrm{O}, 26 \mathrm{~mm} \mathrm{NaHCO}_{3}$, and 10 $\mathrm{mm} \mathrm{D}$-glucose, supplemented with $5 \mu \mathrm{m}$ glutathion and 1 $\mathrm{mm}$ sodium pyruvate. Slices were maintained at room temperature in the same solution until recording.

\section{Electrophysiology}

Whole-cell patch-clamp experiments were performed in a submersion recording chamber under an upright microscope (Ni-E workstation, Nikon). Slices were bathed in ACSF containing $126 \mathrm{~mm} \mathrm{NaCl}, 3 \mathrm{mM} \mathrm{KCl}, 1.25 \mathrm{~mm}$ $\mathrm{NaH}_{2} \mathrm{PO}_{4} \cdot \mathrm{H}_{2} \mathrm{O}, 1.6 \mathrm{~mm} \mathrm{CaCl} \cdot \mathrm{H}_{2} \mathrm{O}, 2 \mathrm{~mm} \mathrm{MgSO}{ }_{4} \cdot 7 \mathrm{H}_{2} \mathrm{O}, 26$ $\mathrm{mm} \mathrm{NaHCO}_{3}$, and $10 \mathrm{~mm}$ D-glucose. M1 Layer $\mathrm{V}$ neurons were visualized with infrared differential interference contrast and fluorescence microscopy (Spectra $\mathrm{X}$ light engine, Lumencor; Froux et al., 2018). PNs were identified on morphologic criteria (triangle-shaped soma) and D2R-positive cells and PV-positive INs (PVINs) were identified by the fluorescence of tdTom. Recording electrodes were pulled from borosilicate glass capillaries (G150-4; Warner Instruments) with a puller (Sutter Instrument, Model P-97) and had a resistance of 5-7 M $\mathrm{M}$. They contained $135 \mathrm{~mm}$ K-gluconate, $3.8 \mathrm{~mm} \mathrm{NaCl}, 1 \mathrm{~mm} \mathrm{MgCl} \cdot 6 \mathrm{H}_{2} \mathrm{O}, 10 \mathrm{~mm}$ HEPES, $0.1 \mathrm{~mm}$ $\mathrm{Na}_{4}$ EGTA, $0.4 \mathrm{~mm} \mathrm{Na}_{2} \mathrm{GTP}$, and $2 \mathrm{~mm}$ MgATP for the current-clamp experiments. For the recordings of spontaneous IPSCs (sIPSCs) and miniature IPSCs (mIPSCs) in voltage clamp experiments, K-gluconate was replaced by $\mathrm{CsCl}$ and $2 \mathrm{~mm}$ Qx-314 was added to prevent action potentials. In all cases, the osmolarity of the intrapipette solution was between 285 and $295 \mathrm{mOsm}$ and $\mathrm{pH}$ was adjusted to 7.2. Experiments were conducted using a Multiclamp 700B amplifier and Digidata 1440 digitizer controlled by Clampex 10.3 (Molecular Devices) at $34^{\circ} \mathrm{C}$. Data were acquired at $20 \mathrm{kHz}$ and low-pass filtered at $4 \mathrm{kHz}$. Whole-cell patch clamp recordings with $\mathrm{CsCl}$ - or K-Glu- filled electrodes were corrected for a junction potential of 4 and $13 \mathrm{mV}$, respectively. In voltage clamp experiments, series resistance was continuously monitored by a step of $-5 \mathrm{mV}$. Data were discarded when the series resistance increased by $>20 \%$. sIPSCs and mIPSCs were recorded at a holding potential of $-64 \mathrm{mV}$.

To evaluate their intrinsic excitability, neurons were injected with increasing depolarizing current pulses (50-pA steps, ranging from 0 to $+550 \mathrm{pA}, 1000$-ms duration). Action potential firing frequency was calculated for each current pulse. To measure the input resistance, a hyperpolarizing $-100 \mathrm{pA}$ pulse current of $1 \mathrm{~s}$ was applied and the voltage response was measured at steady state. Input-output curves (F-I curves, frequency of action potential firing as a function of injected current) were constructed.

\section{Drugs}

Unless otherwise stated, drugs were prepared in distilled water as concentrated stock solutions and stored at $-20^{\circ} \mathrm{C}$. Drugs were diluted daily at the experimental concentrations and perfused in the recording chamber. When indicated, ionotropic glutamatergic and GABAergic transmissions were blocked. NMDA receptors were inhibited by $50 \mu \mathrm{M}$ D-(-)-2-amino-5-phosphonopentanoic acid (APV); AMPA/kainate receptors by $20 \mu \mathrm{M}$ 6,7-dinitroquinoxaline-2,3-dione (DNQX); and $\mathrm{GABA}_{\mathrm{A}}$ receptors by 50 $\mu \mathrm{M}$ picrotoxin. To study sIPSCs or evoked IPSCs (elPSCs), glutamate and $\mathrm{GABA}_{B}$ receptors were blocked by APV, DNQX, and $1 \mu \mathrm{M}(2 \mathrm{~S})-3-[[(1 S)-1$-(1,4-dichlorophenyl)ethyl]amino-2 hydroxypropyl](phenylmethyl) phosphinic acid [CGP55845; dissolved in dimethylsulfoxide (DMSO)]. The D2-like DA receptor agonist (4aR-trans)4,4a,5,6,7,8,8a,9-octahydro-5-propyl-1H-pyrazolo[3,4-g] quino-line hydrochloride (quinpirole, $2 \mu \mathrm{M}$ ) and antagonist (sulpiride, $2 \mu \mathrm{M}$ ) were used. Sulpiride was dissolved in DMSO. Drug effects were measured at least $10 \mathrm{~min}$ after drug perfusion. Chemicals were purchased from Tocris Bioscience, Abcam, or Sigma-Aldrich.

\section{Optogenetics}

To specifically activate PVINs, the cation channelrhodopsin-2 (ChR2) was expressed in PVINs within M1. To this end, the viral vector AAV2.5-EF1a-DIO-hChR2(H134R)EYFP.WPRE.hGH (V2109TI; $6.72 \mathrm{e}^{12} \mathrm{gc} / \mathrm{ml}$; UNC Vector Core) was injected in M1 of PV-Cre:Ai9T mice. Ten mice received three unilateral injections of $0.5 \mu \mathrm{L}$ viral vector solution in $\mathrm{M} 1$ at the following stereotaxic coordinates (from bregma): lateral, 1.125/1.125/1.375 mm, posterior, +1.4/ $+1.15 /+1.4 \mathrm{~mm}$ and depth, $-1.275 /-1.275 /-1.475 \mathrm{~mm}$. The viral vector was pressure-injected using a picospritzer III (Intracel) connected to a glass pipette at a rate of $100 \mu \mathrm{l} /$ $\mathrm{min}$. After the injection, the pipette was left in place for $1 \mathrm{~min}$ before being slowly retracted. Animal were housed for two to three weeks before electrophysiological recordings. An LED-light source ( $473 \mathrm{~nm}, 100 \mathrm{~mW}$; Prizmatix Ltd.) was connected to an optic fiber (Ø: $500 \mu \mathrm{m}$; numeric aperture: 0.63 ) placed close to the region of interest. Single or $10-\mathrm{Hz}$ trains of light pulses of 1-ms duration were used to evoke synaptic transmission between PVIN-expressing ChR2 and PNs.

\section{Experimental design and statistical analysis}

Data analyses were performed with the Clampfit routine, Origin 7, and a custom-made software for the detection and measurement of SIPSCs and mIPSCs (Detection Mini 8.0; Chazalon et al., 2018). To build the cumulative probability distributions, the same number of events $(n=300)$ has been used for all neurons. Statistical analysis was performed with Prism 5 (GraphPad Software). Population data are presented as mean \pm SEM. Paired data were compared using the Wilcoxon signed-rank (WSR) test. Comparisons of F-I relationships were performed with a two-way repeated-measures ANOVA test followed by a Bonferroni test for multiple comparisons (Bichler et al., 2017). The Kolmogorov-Smirnov (K-S) test was used to compare the cumulative distributions. Data were considered statistically significant at $p<0.05$ $(* p<0.05, * * p<0.01, * * * p<0.001$; n.s., not significant).

\section{Results}

\section{Distribution of D2R-expressing cells in the M1 of Drd2-Cre:Ribotag mice}

We took advantage of the Drd2-Cre:Ribotag mice (Puighermanal et al., 2015), which express ribosomal 
protein Rpl22 tagged with the HA epitope selectively in D2R-positive cells, to determine the expression pattern of D2R-positive cells in M1. The analysis of HA-immunoreactivity revealed that $\mathrm{D} 2 \mathrm{R}$-expressing cells are distributed in all cortical layers, with the highest density in Layers II-III ( $\sim 47 \%)$, followed by the deep Layers ( $\mathrm{V}-\mathrm{VI} ; \sim 38 \%)$ and Layer I ( 15\%; Fig. 1A,B). To determine the molecular identity of D2R-expressing cells located in Layers $\mathrm{V}-\mathrm{VI}$ of M1, we performed double immunostaining and quantified the degree of co-localization of HA-immunoreactive neurons with markers of distinct classes of INs (Fig. 1C,D; Ascoli et al., 2008). As illustrated in Figure $1 C$ and quantified in Figure 1D, HA-positive cells mainly correspond to PV-containing INs ( 26\%) and to a lesser extent, Calbindin-D28k (CB)- and Neuropeptide Y (NPY)-positive INs ( $\sim 10 \%$ and $14 \%)$. In contrast, calretinin (CR)/HA colabeled cells represent only $\sim 3 \%$ of HA-positive cells, while neuronal NO synthase (nNOS)/HA neurons were not detected. Although D2R-positive neurons of Layers V-VI might constitute a subpopulation of cortical INs, our results revealed that they largely correspond to PVINs.

\section{Electrophysiological characterization of M1 D2R- expressing cells}

To determine the intrinsic properties of Layer V M1 D2R neurons, whole-cell patch-clamp recordings were performed using ex vivo slices from Drd2-Cre:Ai9T (Fig. 2). We patched neurons in acute brain slices and among the 19 neurons we recorded, three types of D2R-positive neurons were found differing in their electrophysiological properties and the shape of their soma (Fig. 2). 55\% were fast spiking (FS) INs, 30\% were regular spiking non-pyramidal (RSNP) and $15 \%$ were PNs. The FS neurons had a mean resting potential of $-83.86 \pm 2.02 \mathrm{mV}(n=11)$ and were able to fire fast action potentials at a high constant rate. Their action potentials had a short duration and a large afterhyperpolarization (AHP; Fig. 2B, inset) which are general characteristics of FS neurons. The discharge frequency increased as a function of the stimulation intensity and the maximal frequency, measured for high intensities of depolarizing currents ranging from 100 to $230 \mathrm{~Hz}$ (Fig. 2D). Their rheobase differed from one neuron to another and were on average $154.5 \pm 17.13 \mathrm{pA}$. In addition, FS cells had a small input resistance (between 80 and 200 $\mathrm{M} \Omega$, except for a neuron). We performed immunohistochemistry to detect the expression of PV in seven neurons filled with biocytin during whole-cell recording. Six of seven were PV-immunoreactive (data not shown).

The second cell type did not maintain high-frequency repetitive discharges and was classified as RSNP because of the shape of the soma (Fig. 2B, central panel). Action potentials evoked by current injection in RSNP cells had a longer duration and a relatively smaller AHP than those recorded in FS cells. All RSNP cells displayed a resting potential close to $-81.23 \pm 0.93 \mathrm{mV}(n=6)$. They had a low maximal frequency of discharge associated with a low rheobase. At a low discharge frequency, RSNP cells emitted action potentials with moderate or no accommodation.
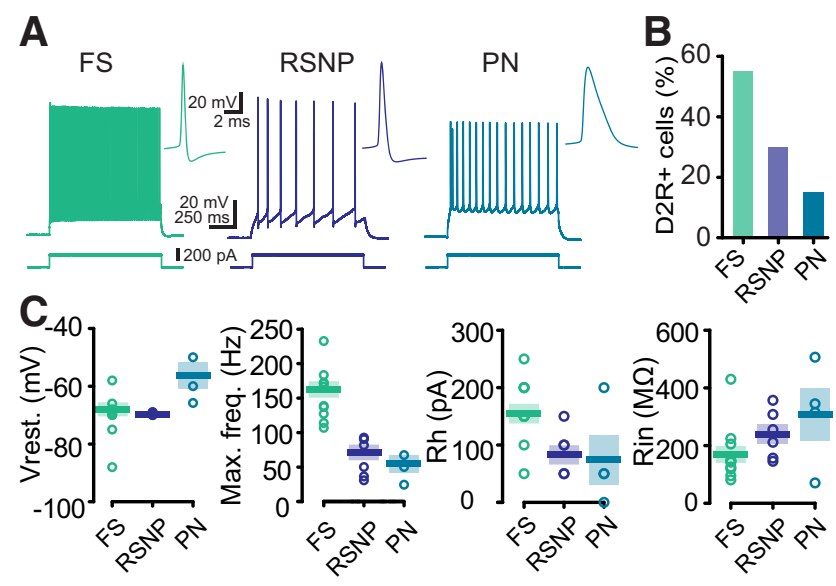

Figure 2. Electrophysiological characterization of D2R-expressing neurons in motor cortex M1 in Drd2-Cre:Ai9T mice. A, Firing behavior of the three types of D2R-expressing neurons in Layer $\mathrm{V}$ of M1. A depolarizing current injection (200 pA, $1 \mathrm{~s})$ evoked a high-frequency spike firing pattern in FS (left) and a lower frequency of discharge in RSNP (middle) and PNs (right). Next to each trace, an expanded view of single spikes and AHP is presented for the three groups of neurons. $\boldsymbol{B}$, Histogram showing the percentage of each type of D2R-expressing neurons in Layer V of M1 $(n=21)$. C , Summary of resting membrane potential (Vrest.), maximal firing frequency (Max. freq.), rheobase (Rh), and input resistance (Rin) in the three cell types.

Finally, a few PNs were identified by the triangular shape of their soma. They exhibited a sustained action potential discharge in response to depolarizing current pulses with a low maximal frequency of discharge (Fig. $2 C)$. PNs had a mean resting potential of $-71.67 \pm$ $6.45 \mathrm{mV}$, a mean input resistance of $309.5 \pm 89.97 \mathrm{M} \Omega$ and a mean rheobase of $75.00 \pm 43.30 \mathrm{pA}(n=4)$.

\section{D2R activation increases the intrinsic excitability of PVINs}

Since the majority of D2R cells recorded in Layer $\mathrm{V}$ of $\mathrm{M} 1$ were FS INs and expressed PV, we switched to the PV-Cre: Ai9T mouse line to focus our study on the PVINs, which are also mainly FS INs (Hu et al., 2014). In PV-Cre:Ai9T brains, PVINs can be easily targeted for recording as they express the fluorescent protein tdTom. We investigated the effect of a typical D2 agonist, quinpirole, on PVINs excitability in M1 Layer V (Fig. 3A). To prevent the influence of spontaneous excitatory and inhibitory inputs on action potential generation, fast glutamatergic and GABAergic transmissions were pharmacologically blocked using DNQX (10 $\mu \mathrm{m}) / \mathrm{D}-\mathrm{AP5}$ (50 $\mu \mathrm{M})$ and picrotoxin $(50 \mu \mathrm{M})$, respectively. Bath application of quinpirole $(2 \mu \mathrm{M})$ changed the intrinsic properties of the PVIN sample. A somatic injection of depolarizing current induced more action potentials in the presence of quinpirole for the same injected current, as exemplified in Figure $3 A$. This was true for all injected currents tested as shown by the frequency/current (F/l) input-output curve (Fig. 3B; $p<0.0001, n=10$; two-way repeated-measures ANOVA). Indeed, quinpirole changed the output-input curve of the 10 PVINs tested, shifting it to the left and thus inducing 

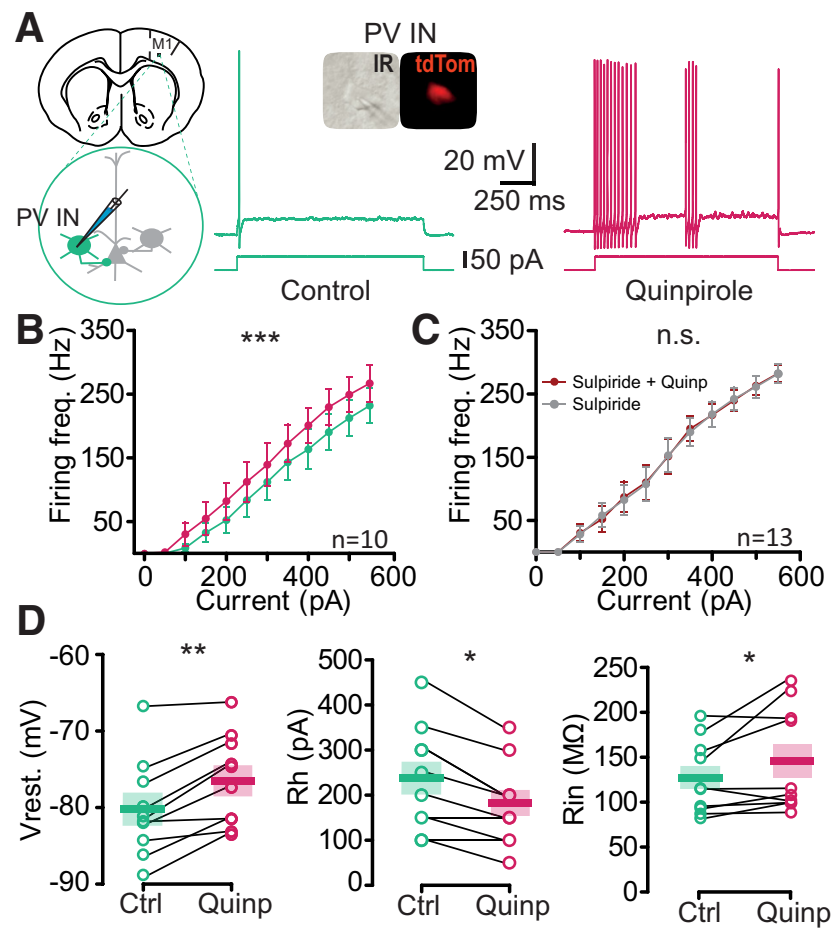

Figure 3. Quinpirole increases the excitability of M1 PVINs. A, left, Schematic of the experiment. PVINs were identified as tdTomato (tdTom in inset) positive neurons in slices from PVCre:Ai9T mouse brain. Representative voltage responses to +50 -pA current injection in a PVIN in control bath solution (green, middle) and after 10 min of perfusion of the D2R agonist quinpirole (Quinp, $2 \mu \mathrm{M}$, red, right). B, Quinpirole enhanced the firing frequency (Firing freq.) of PVINs and significantly shifted the input-output curve to the left $\left(p<0.0001, n=10, F_{(1,96)}=\right.$ 42.64, two-way ANOVA). C, Quinpirole does not increase the firing frequency of PVINs in presence of sulpiride $(p=0.7645$, $n=13, F_{(1,144)}=0.09,011$, two-way ANOVA). Each symbol represents mean \pm SEM. $D$, Summary of the quinpirole effect on resting membrane potential (Vrest.), maximal firing frequency (Max. freq.), rheobase (Rh), and input resistance (Rin; WSR test). The thick bar and the color block represent the mean and the SEM, respectively. GABA, NMDA, and AMPA/kainate receptors were blocked throughout all of the recordings with PTX $(50 \mu \mathrm{M})$, D-AP5 $(50 \mu \mathrm{M})$, and DNQX $(10 \mu \mathrm{M})$, respectively. ${ }^{\star} p<0.05,{ }^{\star \star} p<0.01,{ }^{\star \star \star} p<0.001$; n.s., not significant.

increased excitability. Importantly, the application of the D2R antagonist sulpiride blocked the excitatory effect of quinpirole on PVINs excitability (Fig. 3C). Moreover, quinpirole significantly depolarized the PVINs resting potential from $-80.19 \pm 1.99$ to $-76.44 \pm 1.87 \mathrm{mV}(p=0.002 ; n=$ 10; WSR test), increased their maximal firing frequency $(221.1 \pm 26.9$ to $253.8 \pm 28.7 \mathrm{~Hz}, p=0.002$; WSR test), decreased their rheobase from $235.0 \pm 36.6$ to $180.0 \pm$ $29.1 \mathrm{pA}(p=0.0115$; WSR test), and increased their mean input resistance from $127.3 \pm 12.9$ to $145.4 \pm 18.3 \mathrm{M} \Omega$ $(p=0.0020$; WSR test; Fig. 3D).

\section{D2R activation increases afferent GABAergic synaptic transmission received by $\mathrm{PNs}$}

Since PVINs increased their excitability in the presence of quinpirole, we sought to determine whether in the presence of the D2R agonist, individual PNs in Layer $\mathrm{V}$ received more phasic $\mathrm{GABA}_{\mathrm{A}}$ receptor-mediated inhibition. We first assessed whether quinpirole per se changed the intrinsic properties of PNs. On average, a bath application of quinpirole had no effect, neither on the F/I curve nor on the resting potential or input resistance of the seven PNs recorded (Fig. 4A,B). To determine whether PNs received more GABAergic inhibition, we recorded the IPSCs in PNs, i.e., the sIPSCs and mIPSCs that reflect the action potential-dependent and action potential-independent activities of the inhibitory IN network, respectively. To specifically study the action of quinpirole on sIPSCs and mIPSCs, and to neutralize the potential confounding influence of excitatory and GABA $A_{B}$ neurotransmissions, DNQX $(10 \mu \mathrm{M})$, D-AP5 $(50 \mu \mathrm{M})$, and CGP55845 $(1 \mu \mathrm{M})$ were bathapplied before the perfusion of quinpirole. In these conditions (considered as a control condition), robust sIPSCs were observed in all the recorded PNs at a holding potential of $-64 \mathrm{mV}$, confirming GABAergic inhibitory control of PNs by GABAergic INs (Fig. 4C,F).

The effects of $2 \mu \mathrm{m}$ quinpirole on sIPSCs were studied on 10 neurons. Quinpirole increased the amplitude (Fig. $4 E$ ) without changing the frequency of the sIPSCs (Fig. $4 D$ ). Indeed, the cumulative probabilities of the frequency of sIPSCs in the control and the quinpirole groups were similar (Fig. 4D; $p>0.05$, K-S test). However, the cumulative probability of the amplitude of the sIPSCs showed an increase in the quinpirole group (Fig. $4 E ; p<0.0001$, K-S test) compared with the control group. Moreover, quinpirole significantly increased the decay time from 7.68 \pm 0.67 to $9.07 \pm 0.75 \mathrm{~ms}$ ( $p=0.059, n=10$; WSR test). Next, we examined the effect of quinpirole in PNs in the presence of $1 \mu \mathrm{m}$ TTX, to isolate mIPSCs (Fig. 4F). As for sIPSCs, analysis of the cumulative probability (Fig. $4 G, H$ ) with the K-S test revealed that D2R activation increased mIPSC amplitude with no effect on their frequency. The decay time of IPSC was significantly increased from $7.66 \pm 0.65$ to $8.88 \pm 0.70 \mathrm{~ms}(p=0.0273, n=10 ;$ WSR test). The frequency was unchanged on average, but it is important to note that quinpirole had a variable effect on individual neurons.

\section{D2R activation enhances GABAergic transmission at PVIN-PN synapses}

Our results on GABAergic IPSCs suggested that D2R activation by quinpirole induced more activity in the inhibitory network. However, the increase observed may be due to any type of inhibitory IN. To determine whether quinpirole changes synaptic transmission between PVINs and PNs, we used optogenetics to selectively study PVIN-PN synapse properties (Fig. 5). We expressed the channelrhodopsin ChR2 in PVINs via local viral transfection in M1 of PV-Cre:Ai9T mice using an AAV2.5-EF1aDIO-hChR2(H134R)-EYFP vector (Fig. 5A). We used 473$\mathrm{nm}$ light flashes to stimulate PVINs while recording from PNs. We first confirmed that $1 \mathrm{~ms}$ flashes of light were able to reliably trigger action potentials in PVINs. As illustrated by the raster plot in Figure $5 B$, each flash in the train evoked one or two action potentials in the transfected PVIN. In a second step, we recorded the optically- 
A
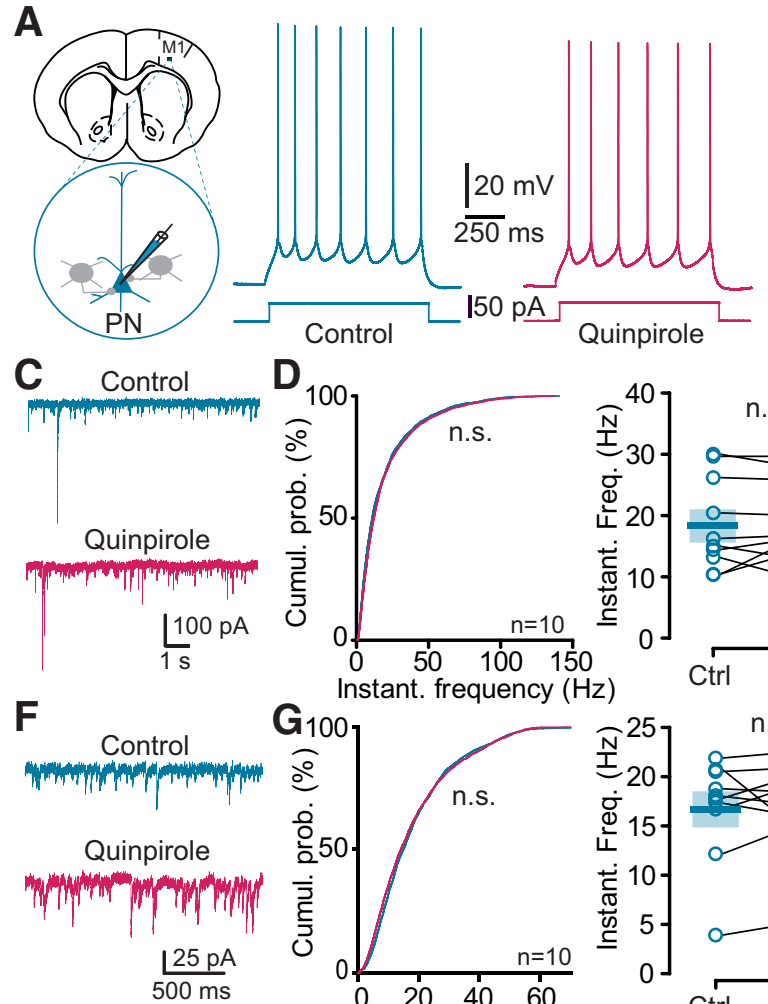

$\mathbf{G}_{1}$ Instant. frequency $(\mathrm{Hz}$

G100

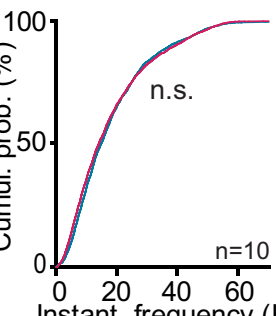

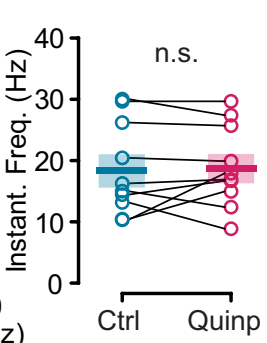

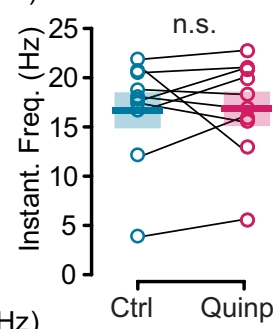

B
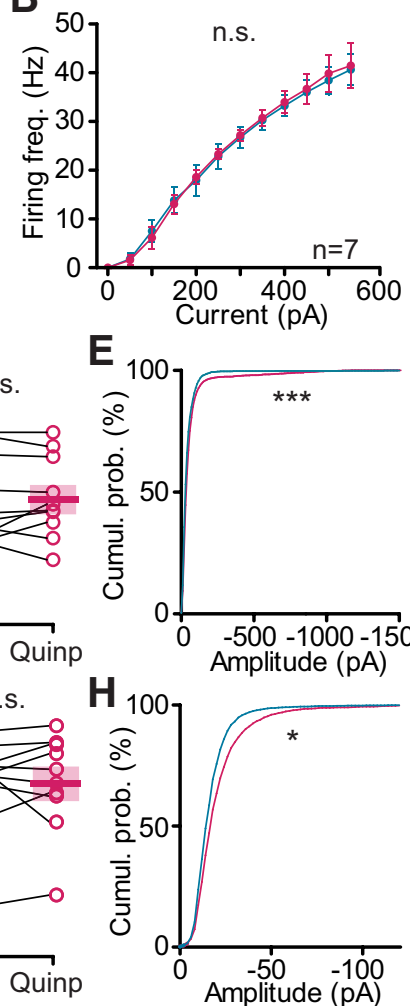
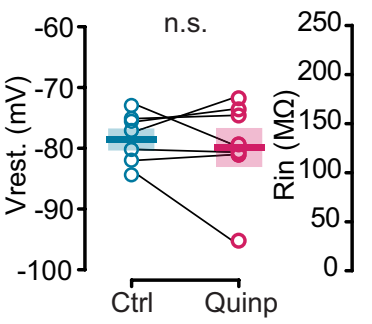

n.s.
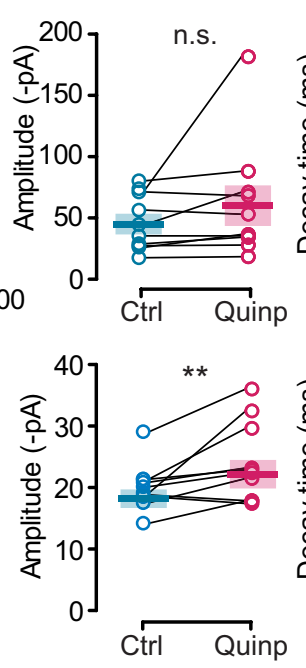
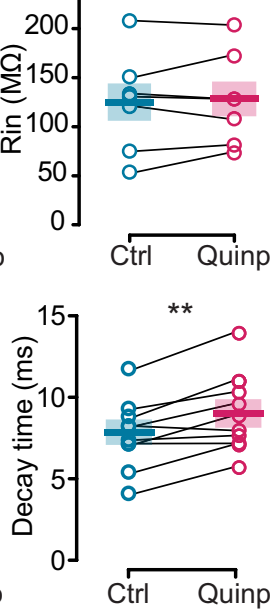

n.s.

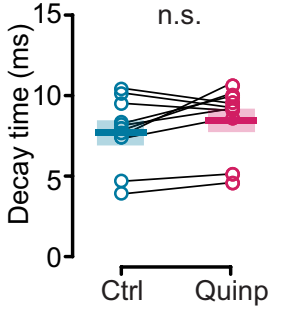

Figure 4. Effect of quinpirole on the electrical activity and sIPSCs (sIPSCs and mIPSCs) of PNs. A, Schematic of the experiment. PNs were identified by their morphology, the absence of tdTomato in their soma as well as their intrinsic properties when possible. Example of voltage responses to +50 -pA current injection recorded in a representative PN in control (left, blue) and in quinpirole (right, red). B, Quinpirole did not change the firing frequency of PNs $\left(p=0.6453, n=7, F_{(1,72)}=0.21\right.$, two-way ANOVA), nor the resting potential or input resistance $(p=1.000$ and $p=0.8982$, respectively; $n=7$, WSR test). $\boldsymbol{C}$, Representative traces of sIPSCs recorded from a M1 PN (left) in control conditions (top trace, blue) and in the presence of quinpirole (bottom trace, red). $\boldsymbol{D}$, Cumulative distribution (left) and mean (right) of sIPSC instantaneous frequency in the control (blue) and in the presence of quinpirole (red). No differences were observed ( $p=0.9987$, K-S test and $p>0.9999$, WSR test). $E$, Cumulative distribution (left) and mean (middle) of sIPSC amplitude and value of $\tau$ (right). Note that cumulative distribution of the amplitude and decay time differed significantly between control and quinpirole conditions ( $p<0.0001, \mathrm{~K}-\mathrm{S}$, and $p=0.0059$, WSR test, $n=10)$. $\boldsymbol{F}-\boldsymbol{H}$, Same representations as in $\boldsymbol{C}-\boldsymbol{E}$ for mIPSCs. Note that similarly to sIPSCs, only the cumulative distribution $(p<0.05$, K-S test) and mean of mIPSC amplitude differed significantly between control and quinpirole conditions ( $p=0.0098 ; n=10$, WSR test). ${ }^{*} p<0.05,{ }^{* *} p<0.01,{ }^{* * *} p<0.001$; n.s., not significant.

evoked IPSCs from PNs (Fig. 5C). PNs were identified as described previously (Fig. 4) and displayed a PN-typical firing pattern on depolarizing current steps (Fig. 5C). Light flashes reliably elicited eIPSCs in PNs, which were potentiated by bath application of $2 \mu \mathrm{M}$ quinpirole (Fig. $5 D$ ), increasing their mean amplitude from $280.3 \pm 68.52$ to $321.6 \pm 75.67 \mathrm{pA}(p=0.0371, n=10$; WSR test). This result strongly suggested that GABAergic synaptic transmission between PVINs and PNs was enhanced by quinpirole. We further characterized the short-term plasticity of the PVINs-PNs synapses using 10 flashes of $1 \mathrm{~ms}$ at $10 \mathrm{~Hz}$; Fig. 5E). The inhibitory inputs to PNs showed pronounced synaptic short-term depression, but bath-applied quinpirole did not change the profile of synaptic transmission, which remained depressed (Fig. 5F).

\section{Discussion}

In the present study, we first performed in mice a quantitative mapping of $M 1$ neuronal populations expressing D2R. These neurons are largely present in Layers II/III and
Layer V, and are mainly PVINs in Layer V, based on immunochemistry and electrophysiological characterization. Then, combining electrophysiology and optogenetics, we demonstrated ex vivo that the activation of D2R robustly increases the excitability of PVINs and enhances the synaptic transmission between PVINs and PNs.

\section{D2R-expressing cells in Layer $\mathbf{V}$ of $\mathbf{M} 1$}

Previous studies have shown that M1 cortical neurons express both D1 and D2 classes of DA receptors (Lidow et al., 1989; Seamans and Yang, 2004) and receive direct DA projections from VTA and SNc via meso-cortical pathways (Descarries et al., 1987). For many years, cortical D2R has been a focus of interest because of its involvement in many cognitive functions initiated or modulated by DA. However, the relatively low expression of cortical D2R makes its detection very difficult. Consequently, it is more difficult to identify the nature of the neurons expressing D2R, a difficulty further amplified by the massive heterogeneity of neurons. Most studies of cortical D2R 
A AAV.DIO.ChR2.EYFP

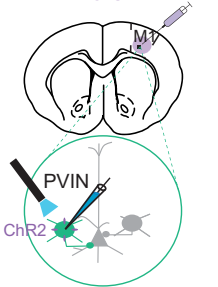

C

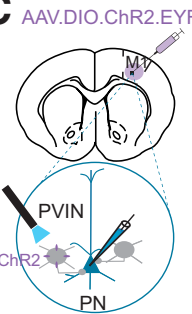

E

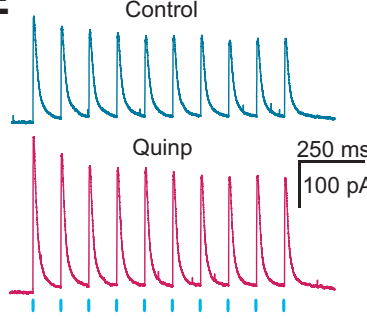

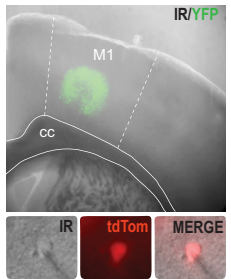

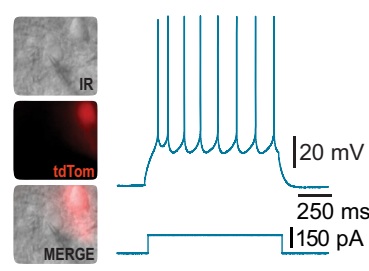

B

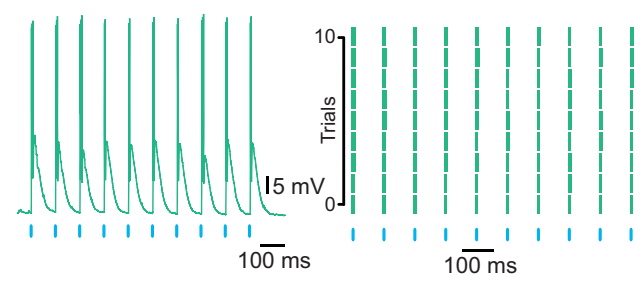

D

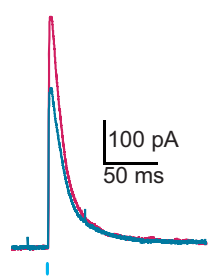

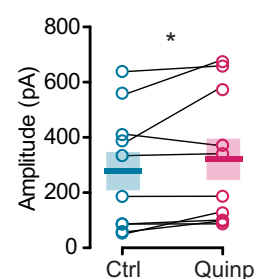

$\mathbf{F}$
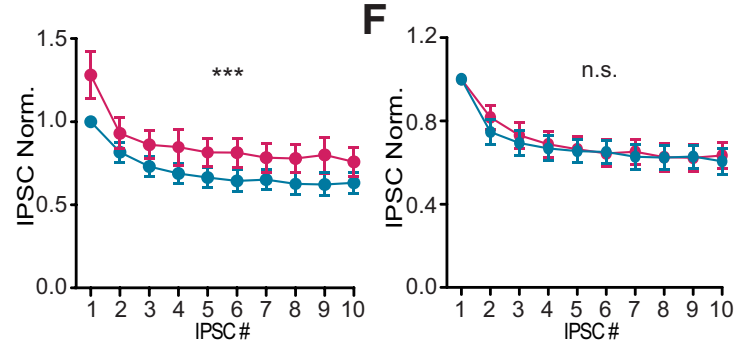

Figure 5. Quinpirole increases GABAergic synaptic transmission at the PVIN-PN synapse. A, Schematic of the experiment. An AAV. DIO.ChR2.EYFP virus was injected in M1 two weeks before ex vivo recordings. Representative slice showing the expression of ChR2-EYFP in M1. PVINs (tdTom-positive) were patched to verify our ability to manipulate their activity. B, Light reliably induced action potentials in a PVIN (left). Each flash of light (blue line) evoked one or two action potentials in PVINs as presented in an example (left) of an intracellularly recorded PVIN and in the raster plot of spiking in different trials (right). Each blue tick represents a flash of light $(473 \mathrm{~nm}, 1 \mathrm{~ms})$ and each green tick represents a spike. C, Schematic of the recording configuration from a postsynaptic PN during photoactivation of PVINs (left). Soma of PNs had a triangular shape, were tdTom-negative and PV-negative (middle). Representative firing pattern of recorded PNs to a 150-pA, 500-ms current step (blue, right). $\boldsymbol{D}$, Sample traces and quantification of light-evoked IPSCs recorded in the same PN before (blue, control trace) and after 10 min of quinpirole perfusion (red). Mean of the amplitude of the evoked response $(p=0.0371, n=10$, WSR). Mean and SEM are represented. $\boldsymbol{E}$, Sample traces and quantification of responses to repetitive photostimulation $(10 \mathrm{~Hz})$ recorded before (blue) and after bath application of quinpirole (red). Photoactivation of PVINs produced large initial IPSCs that depress rapidly. In the graph, the IPSC amplitudes were normalized to that of the first IPSC in the control condition for each neuron recorded $\left(p<0.0001, n=10, F_{(1,180)}=19.36\right)$. $\boldsymbol{F}$, Short-term synaptic dynamics of the elPSCs in PNs induced by the photoactivation of PVINs were not changed in presence of quinpirole. IPSC amplitudes were normalized to the first IPSC of the train in each condition $\left(p=0.1563, n=10, F_{(1,180)}=0.4749\right)$. ${ }^{*} p<0.05$, ${ }^{* \star} p<0.001$; n.s., not significant.

have focused on the prefrontal cortex and several studies have detected the presence of D2R mRNA in PFC by in situ hybridization, revealing its expression in PNs and minor subtypes of INs (Gaspar et al., 1995). Recently, technical limitations were overcome with a highly sensitive and multimodal approach to map cortical D2R-expressing neurons (Drd2-Cre:Ribotag mouse), which has allowed the identification of previously uncharacterized clusters of D2R-expressing neurons in limbic and sensory regions of the adult mouse brain (Khlghatyan et al., 2018). Unfortunately, the authors did not perform quantitative mapping of the M1 neuronal populations expressing D2R. In the present study, using both the Drd2-Cre:Ribotag and Drd2-Cre:Ai9T mouse lines, we show that D2R-expressing cells are distributed in all cortical layers of M1 and broadly expressed in Layer $\mathrm{V}$. The molecular characterization of D2R-expressing cells in Layer $\mathrm{V}$ revealed a majority of PVINs and to a lesser extent, populations of
CB- and NPY-positive cells. Electrophysiological characterization in the Drd2-Cre:Ai9T mouse line revealed three main classes of neurons expressing D2R in Layer V of M1: FS, RSNP and PN. The majority of the D2R-expressing cells are FS neurons, which are also mainly PV-positive neurons. Since PVINs account for a quarter of the D2Rpositive neurons, they are likely to play a specific role, still unknown, as a target for the DA modulation of cortical microcircuits in M1.

\section{D2R modulation of intrinsic excitability of PVINs in M1}

Although dopaminergic fibers and DA receptors in M1 have been clearly demonstrated (Descarries et al., 1987; Hosp et al., 2011), their functional significance remains poorly understood. Conflicting evidence indicates excitatory and inhibitory effects on electrical activity in vivo (Vitrac and Benoit-Marand, 2017). It has been shown that 
in pyramidal cells, D2R activation mediates inhibition occurring via postsynaptic inhibition of PKA and activation of PLC-IP3 and intracellular $\mathrm{Ca}^{2+}$. Most studies have not tested specific cell types and there is no data available regarding DA modulation of specific subpopulations of INs in M1. In this study, we focused on PVINs of Layer V. It has been shown that PVINs in the motor cortex receives direct inputs from the VTA (Duan et al., 2020). We found that activating D2R caused the depolarization and an increase in the excitability of most of the PVINs recorded, even if an interindividual variability of the effect was observed. The excitatory effect of the D2R agonist quinpirole on INs has been already observed on INs in prefrontal cortex (PFC) slices from adult mice (Tseng and O'Donnell, 2006). As cell excitability was determined by assessing the response to intracellular injection alone and in the presence of fast synaptic blockers, this is likely to reflect the postsynaptic effects of the agonist and not a modulation of pre-synaptic afferents. However, further studies will be required to determine whether this excitatory effect reflects a direct D2R postsynaptic action on PVINs as observed in PFC (Tseng and O'Donnell, 2004). Such excitatory effect of D2R stimulation could be explained by the downstream $\beta$-arrestin 2 signaling (Urs et al., 2016), or activation of D2R autoreceptors and release of the co-transmitter neurotensin, which is present in a subpopulation of DA neurons from the VTA projecting to PFC (Petrie et al., 2005) that may also project to $\mathrm{M} 1$.

\section{D2R modulation of GABAergic synaptic transmission in M1}

Proper brain function depends on a correct balance between excitatory and inhibitory signaling (Markram et al., 2015) and PVINs are crucial for such network functionality. Indeed, they exert powerful actions on cortical network activity by contributing to feedback and feedforward inhibition of PNs (Hu et al., 2014). To determine whether the D2R agonist quinpirole modulates the afferent GABAergic synaptic transmission to PN in M1, we examined the IPSCs in PNs. Measurement of the changes in sIPSCs and mIPSCs are a sensitive means to estimate the locus of a drug effect. Typically, changes in IPSC amplitudes are associated with a postsynaptic site of modulator action, whereas changes in IPSC frequency are likely to be due to an interaction with a presynaptic site that changes the probability of transmitter release (Lupica, 1995). Since the excitability of PVINs was increased by quinpirole, we expected an increase in IPSC frequency, but this was not the case. Here, quinpirole increased the amplitude of both sIPSCs and mIPSCs recorded in PNs with no effect on their frequency. One possible explanation is that since the PVINs are not spontaneously firing at their resting potential, the $5 \mathrm{mV}$ depolarization generated by quinpirole may not be large enough to raise the resting membrane potential to the spike threshold in the absence of excitatory transmission. Another possible explanation is the various origins of the GABAergic IPSCs. We studied all the GABAergic inhibitory currents received by PNs and cannot exclude an effect of quinpirole on other GABAergic
INs that may mask the effect on frequency, or this may occur only in a subset of PVIN-PN synapses. Finally, the effect could be more complex and can combine a presynaptic and postsynaptic effects of DA.

\section{D2R modulation of PVINs-PN synaptic transmission in M1}

In order to be specific to PVIN-PNs synapses and to overcome the fact that these PVINs do not spontaneously fire in slices, we used optogenetics. Our results show that bath application of quinpirole potentiated the optically-evoked elPSCs in PNs. Using a $10-\mathrm{Hz}$ train of stimulations, we showed an adaptive depression of this synapse from the second optical stimulation that persisted with quinpirole. These observations suggest that quinpirole mainly acts on postsynaptic sites and show that the adaptive depression is maintained. It is also possible that quinpirole-induced depolarization of PVINs membrane potential allows the recruitment of a greater number of neurons during light activation, which can account for the increase in optically-evoked IPSC amplitude without changes in the depression profile. Finally, bath application of quinpirole clearly modulates the intrinsic properties of PVINs. However, as the timing of DA release is critical for plasticity induction (Yagishita et al., 2014), investigating how endogenous DA release controls PVIN-PN GABAergic synaptic transmission and plasticity using optogenetics is crucial.

\section{Functional implications}

M1 is particularly important in acquisition and maintenance of motor skills and is a central locus for motor learning. Indeed, pharmacological or optogenetic inactivation of M1 is highly effective in reducing motor aptitude (Peters et al., 2014; Guo et al., 2015; Otchy et al., 2015). The lesion of M1 before training abolishes the ability to learn stereotyped movements but does not impair the execution of an already learned motor skill (Guo et al., 2015; Kawai et al., 2015), demonstrating a role for M1 in "tutoring" subcortical circuits during skill learning. Moreover, recent studies have shown that DA plays a key role in motor learning and memory in M1 (MolinaLuna et al., 2009; Leemburg et al., 2018), particularly in spine regulation and synaptic plasticity (Xu et al., 2009; Guo et al., 2015). Interestingly, it has been recently shown that PVINs exhibit a gradual increase in axonal boutons during motor training (Chen et al., 2015). As we show that the activity of PVINs can be modulated by activation of D2R in M1, these data suggest that PVINs and D2R may be crucial for learning sophisticated motor sequences. Interestingly, it has been shown that striatal PVINs enhance behavioral performance in a reward-conditioning task, but their contribution declines as learning progresses (Lee et al., 2017), suggesting dynamic involvement during the learning of the task. Thus, we expect that following the loss of DA in M1 in conditions such as Parkinson's disease, plasticity of PVINs in M1 will be altered and can lead to the cognitive deficits observed in this pathology. 


\section{References}

Ascoli GA, Alonso-Nanclares L, Anderson SA, Barrionuevo G, Benavides-Piccione R, Burkhalter A, Buzsáki G, Cauli B, Defelipe J, Fairén A, Feldmeyer D, Fishell G, Fregnac Y, Freund TF, Gardner D, Gardner EP, Goldberg JH, Helmstaedter M, Hestrin S, Karube $F$, et al. (2008) Petilla terminology: nomenclature of features of GABAergic interneurons of the cerebral cortex. Nat Rev Neurosci 9:557-568.

Awenowicz PW, Porter LL (2002) Local application of dopamine inhibits pyramidal tract neuron activity in the rodent motor cortex. $J$ Neurophysiol 88:3439-3451.

Beaulieu JM, Gainetdinov RR (2011) The physiology, signaling, and pharmacology of dopamine receptors. Pharmacol Rev 63:182217.

Berke JD (2018) What does dopamine mean? Nat Neurosci 21:787793.

Bichler EK, Elder CC, García PS (2017) Clarithromycin increases neuronal excitability in CA3 pyramidal neurons through a reduction in GABAergic signaling. J Neurophysiol 117:93-103.

Chazalon M, Paredes-Rodriguez E, Morin S, Martinez A, CristóvãoFerreira S, Vaz S, Sebastiao A, Panatier A, Boué-Grabot E, Miguelez C, Baufreton J (2018) GAT-3 dysfunction generates tonic inhibition in external globus pallidus neurons in parkinsonian rodents. Cell Rep 23:1678-1690.

Chen SX, Kim AN, Peters AJ, Komiyama T (2015) Subtype-specific plasticity of inhibitory circuits in motor cortex during motor learning. Nat Neurosci 18:1109-1115.

Courtin J, Chaudun F, Rozeske RR, Karalis N, Gonzalez-Campo C, Wurtz H, Abdi A, Baufreton J, Bienvenu TCM, Herry C (2014) Prefrontal parvalbumin interneurons shape neuronal activity to drive fear expression. Nature 505:92-96.

Dawson TM, Gehlert DR, Wamsley JK (1986) Quantitative autoradiographic localization of central dopamine D-1 and D-2 receptors. Adv Exp Med Biol 204:93-118.

DeFelipe J, López-Cruz PL, Benavides-Piccione R, Bielza C, Larrañaga $P$, Anderson $S$, Burkhalter $A$, Cauli $B$, Fairén $A$, Feldmeyer D, Fishell G, Fitzpatrick D, Freund TF, González-Burgos G, Hestrin S, Hill S, Hof PR, Huang J, Jones EG, Kawaguchi Y, et al. (2013) New insights into the classification and nomenclature of cortical GABAergic interneurons. Nat Rev Neurosci 14:202-216.

Descarries L, Lemay B, Doucet G, Berger B (1987) Regional and laminar density of the dopamine innervation in adult rat cerebral cortex. Neuroscience 21:807-824.

Donato F, Rompani SB, Caroni P (2013) Parvalbumin-expressing basket-cell network plasticity induced by experience regulates adult learning. Nature 504:272-276.

Duan Z, Li A, Gong H, Li X (2020) A whole-brain map of long-range inputs to GABAergic interneurons in the mouse caudal forelimb area. Neurosci Bull. Advance online publication. Retrieved Jan 19, 2020. doi: 10.1007/s12264-019-00458-6.

Duvarci S, Simpson EH, Schneider G, Kandel ER, Roeper J, Sigurdsson T (2018) Impaired recruitment of dopamine neurons during working memory in mice with striatal D2 receptor overexpression. Nat Commun 9:2822.

Estebanez L, Hoffmann D, Voigt BC, Poulet JFA (2017) Parvalbuminexpressing GABAergic neurons in primary motor cortex signal reaching. Cell Rep 20:308-318.

Franklin K, Paxinos G (2007) The mouse brain in stereotaxic coordinates, Ed 3. San Diego: Elsevier Academic Press.

Froux L, Le Bon-Jego M, Miguelez C, Normand E, Morin S, Fioramonti S, Barresi M, Frick A, Baufreton J, Taupignon A (2018) D5 dopamine receptors control glutamatergic AMPA transmission between the motor cortex and subthalamic nucleus. Sci Rep 8:8858.

Gaspar P, Bloch B, Le Moine C (1995) D1 and D2 receptor gene expression in the rat frontal cortex: cellular localization in different classes of efferent neurons. Eur J Neurosci 7:1050-1063.

Guo L, Xiong H, Kim JI, Wu YW, Lalchandani RR, Cui Y, Shu Y, Xu T, Ding JB (2015) Dynamic rewiring of neural circuits in the motor cortex in mouse models of Parkinson's disease. Nat Neurosci 18:1299-1309.

Herbison AE, Simonian SX, Norris PJ, Emson PC (1996) Relationship of neuronal nitric oxide synthase immunoreactivity to $\mathrm{GnRH}$ neurons in the ovariectomized and intact female rat. J Neuroendocrinol 8:73-82.

Hosp JA, Luft AR (2013) Dopaminergic meso-cortical projections to M1: role in motor learning and motor cortex plasticity. Front Neurol 4:15.

Hosp JA, Nolan HE, Luft AR (2015) Topography and collateralization of dopaminergic projections to primary motor cortex in rats. Exp Brain Res 233:1365-1375.

Hosp JA, Pekanovic A, Rioult-Pedotti MS, Luft AR (2011) Dopaminergic projections from midbrain to primary motor cortex mediate motor skill learning. J Neurosci 31:2481-2487.

Hu H, Gan J, Jonas P (2014) Fast-spiking, parvalbumin + GABAergic interneurons: from cellular design to microcircuit function. Science 345:1255263.

Kaiser T, Ting JT, Monteiro P, Feng G (2016) Transgenic labeling of parvalbumin-expressing neurons with tdTomato. Neuroscience 321:236-245.

Kawai R, Markman T, Poddar R, Ko R, Fantana AL, Dhawale AK, Kampff AR, Ölveczky BP (2015) Motor cortex is required for learning but not for executing a motor skill. Neuron 86:800-812.

Khlghatyan J, Quintana C, Parent M, Beaulieu JM (2018) High sensitivity mapping of cortical dopamine D2 receptor expressing neurons. Cereb Cortex 29:3813-3827.

Lee K, Holley SM, Shobe JL, Chong NC, Cepeda C, Levine MS, Masmanidis SC (2017) Parvalbumin interneurons modulate striatal output and enhance performance during associative learning. Neuron 93:1451-1463.

Leemburg S, Canonica T, Luft A (2018) Motor skill learning and reward consumption differentially affect VTA activation. Sci Rep 8:687.

Lewis DA, Campbell MJ, Foote SL, Goldstein M, Morrison JH (1987) The distribution of tyrosine hydroxylase-immunoreactive fibers in primate neocortex is widespread but regionally specific. J Neurosci 7:279-290.

Lidow MS, Goldman-Rakic PS, Rakic P, Innis RB (1989) Dopamine D2 receptors in the cerebral cortex: distribution and pharmacological characterization with $[3 \mathrm{H}]$ raclopride. Proc Natl Acad Sci USA 86:6412-6416.

Lodato S, Shetty AS, Arlotta P (2015) Cerebral cortex assembly: generating and reprogramming projection neuron diversity. Trends Neurosci 38:117-125.

Lupica CR (1995) Delta and mu enkephalins inhibit spontaneous GABA-mediated IPSCs via a cyclic AMP-independent mechanism in the rat hippocampus. J Neurosci 15:737-749.

Markram H, Muller E, Ramaswamy S, Reimann MW, Abdellah M, Sanchez CA, Ailamaki A, Alonso-Nanclares L, Antille N, Arsever S, Kahou GAA, Berger TK, Bilgili A, Buncic N, Chalimourda A, Chindemi G, Courcol JD, Delalondre F, Delattre V, Druckmann S, et al. (2015) Reconstruction and simulation of neocortical microcircuitry. Cell 163:456-492.

Molina-Luna K, Pekanovic A, Röhrich S, Hertler B, Schubring-Giese M, Rioult-Pedotti M-S, Luft AR (2009) Dopamine in motor cortex is necessary for skill learning and synaptic plasticity. PLoS One 4: e7082.

Nieoullon A (2002) Dopamine and the regulation of cognition and attention. Prog Neurobiol 67:53-83.

Otchy TM, Wolff SBE, Rhee JY, Pehlevan C, Kawai R, Kempf A, Gobes SMH, Ölveczky BP (2015) Acute off-target effects of neural circuit manipulations. Nature 528:358-363.

Peters AJ, Chen SX, Komiyama T (2014) Emergence of reproducible spatiotemporal activity during motor learning. Nature 510:263267.

Petrie KA, Schmidt D, Bubser M, Fadel J, Carraway RE, Deutch AY (2005) Neurotensin activates GABAergic interneurons in the prefrontal cortex. J Neurosci 25:1629-1636. 
Puighermanal E, Biever A, Espallergues J, Gangarossa G, De Bundel $D$, Valjent $E$ (2015) drd2-cre:ribotag mouse line unravels the possible diversity of dopamine d2 receptor-expressing cells of the dorsal mouse hippocampus. Hippocampus 25:858-875.

Schultz W (2007) Multiple dopamine functions at different time courses. Annu Rev Neurosci 30:259-288.

Seamans JK, Yang CR (2004) The principal features and mechanisms of dopamine modulation in the prefrontal cortex. Prog Neurobiol 74:1-58.

Tseng KY, O'Donnell P (2004) Dopamine-glutamate interactions controlling prefrontal cortical pyramidal cell excitability involve multiple signaling mechanisms. J Neurosci 24:5131-5139.

Tseng KY, O'Donnell P (2006) Dopamine modulation of prefrontal cortical interneurons changes during adolescence. Cereb Cortex 17:1235-1240.

Urs NM, Gee SM, Pack TF, McCorvy JD, Evron T, Snyder JC, Yang X, Rodriguiz RM, Borrelli E, Wetsel WC, Jin J, Roth BL, O'Donnell P, Caron MG (2016) Distinct cortical and striatal actions of a $\beta$-arrestin-biased dopamine D2 receptor ligand reveal unique antipsychotic-like properties. Proc Natl Acad Sci USA 113: E8178-E8186.

Vitrac C, Benoit-Marand M (2017) Monoaminergic modulation of motor cortex function. Front Neural Circuits 11:72.

Vitrac C, Péron S, Frappé I, Fernagut PO, Jaber M, Gaillard A, Benoit-Marand M (2014) Dopamine control of pyramidal neuron activity in the primary motor cortex via D2 receptors. Front Neural Circuits 8:13.

Weiner DM, Levey AI, Sunahara RK, Niznik HB, O’Dowd BF, Seeman P, Brann MR (1991) D1 and D2 dopamine receptor mRNA in rat brain. Proc Natl Acad Sci USA 88:1859-1863.

Xu T, Yu X, Perlik AJ, Tobin WF, Zweig JA, Tennant K, Jones T, Zuo $Y$ (2009) Rapid formation and selective stabilization of synapses for enduring motor memories. Nature 462:915-919.

Yagishita S, Hayashi-Takagi A, Ellis-Davies GCR, Urakubo H, Ishii S, Kasai $H$ (2014) A critical time window for dopamine actions on the structural plasticity of dendritic spines. Science 345:1616-1620. 